\title{
Pulmonary physiology is poorly associated with radiological extent of disease in systemic sclerosis-associated interstitial lung disease
}

\author{
To the Editor:
}

Trials in systemic sclerosis-associated interstitial lung disease (SSc-ILD) typically use forced vital capacity (FVC) as the primary end-point [1, 2]; however, pulmonary function tests have limitations in SSc-ILD, notably being influenced by non-ILD factors such as pulmonary hypertension and diseases of the chest wall. High-resolution computed tomography (HRCT) may provide a more direct assessment of ILD severity, but HRCT-derived visual and quantitative scores also have limitations. The objective of this study was to determine the correlation of visual HRCT findings with severity, progression and mortality of SSc-ILD, and specifically whether HRCT findings and lung function measurements provide redundant or complementary measures of SSc-ILD severity and prognosis.

Patients with SSc-ILD were recruited from 1997 to 2014 from an ILD clinic at the University of British Columbia (UBC) with a separate validation cohort from the University of California San Francisco (UCSF). All patients had fibrotic ILD on HRCT and satisfied American College of Rheumatology diagnostic criteria for SSc [3, 4]. There were no exclusion criteria. All patients provided written informed consent for participation in prospective ILD databases (UBC H14-02858, UCSF \#10-01592).

HRCTs were visually scored by one of five experienced chest radiologists (four at UBC, one at UCSF), each with >5 years' experience. HRCTs for the UBC cohort were performed at diagnosis and approximately every 3 years thereafter until death or censoring, and baseline HRCTs were available for scoring from the UCSF cohort. For the UBC cohort, HRCT scoring was based on three anatomical zones for each lung [5]. Honeycombing, ground glass (either isolated or admixed with fibrosis) and reticulation were recorded as percentage of affected lung in each zone, rounded to the nearest $5 \%$. HRCT fibrosis score was calculated as the combined percentage of honeycombing and reticulation, and total ILD score also including the percentage of ground glass. UCSF scoring was based on five anatomical zones for each lung with determination of the HRCT total ILD score [6]. Pulmonary function tests were performed as clinically indicated (typically every 6 months). The SADL and ILD-GAP models were calculated as previously described $[7,8]$.

Linear regression was used to determine the association of HRCT scores with baseline lung function. For survival, Cox proportional hazards analysis was performed unadjusted and then with two separate models that adjusted for the SADL and ILD-GAP models. A linear mixed effects model was used to determine association of HRCT scores with change in FVC and diffusing capacity of the lung for carbon monoxide (DLCO) in the 1.5 years preceding and following each HRCT, allowing patients with multiple HRCTs to contribute non-overlapping lung function trajectories that corresponded to the date of each HRCT. The UCSF cohort was used for validation of the analysis examining the correlation of HRCT scores with baseline lung function.

A total of 394 HRCT scans were analysed from 145 UBC patients (84\% female) and 89 HRCTs from 89 UCSF patients $(72 \%$ female), with a pooled mean age of 54 years, FVC of $75 \%$, and DLCO of $53 \%$ at diagnosis. In the UBC cohort, $27 \%$ of patients had a $\geqslant 10$ pack-year smoking history, $98 \%$ had antinuclear antibodies, $37 \%$ had anti-Scl-70 antibodies, and 14\% had anti-centromere antibodies. A right ventricular

@ERSpublications

Pulmonary physiology is poorly associated with radiological extent of disease in systemic sclerosisassociated interstitial lung disease http://ow.ly/Bx0X30o92aV

Cite this article as: Jhajj A, Gill HP, Hague CJ, et al. Pulmonary physiology is poorly associated with radiological extent of disease in systemic sclerosis-associated interstitial lung disease. Eur Respir J 2019; 53 : 1802182 [https://doi.org/10.1183/13993003.02182-2018]. 


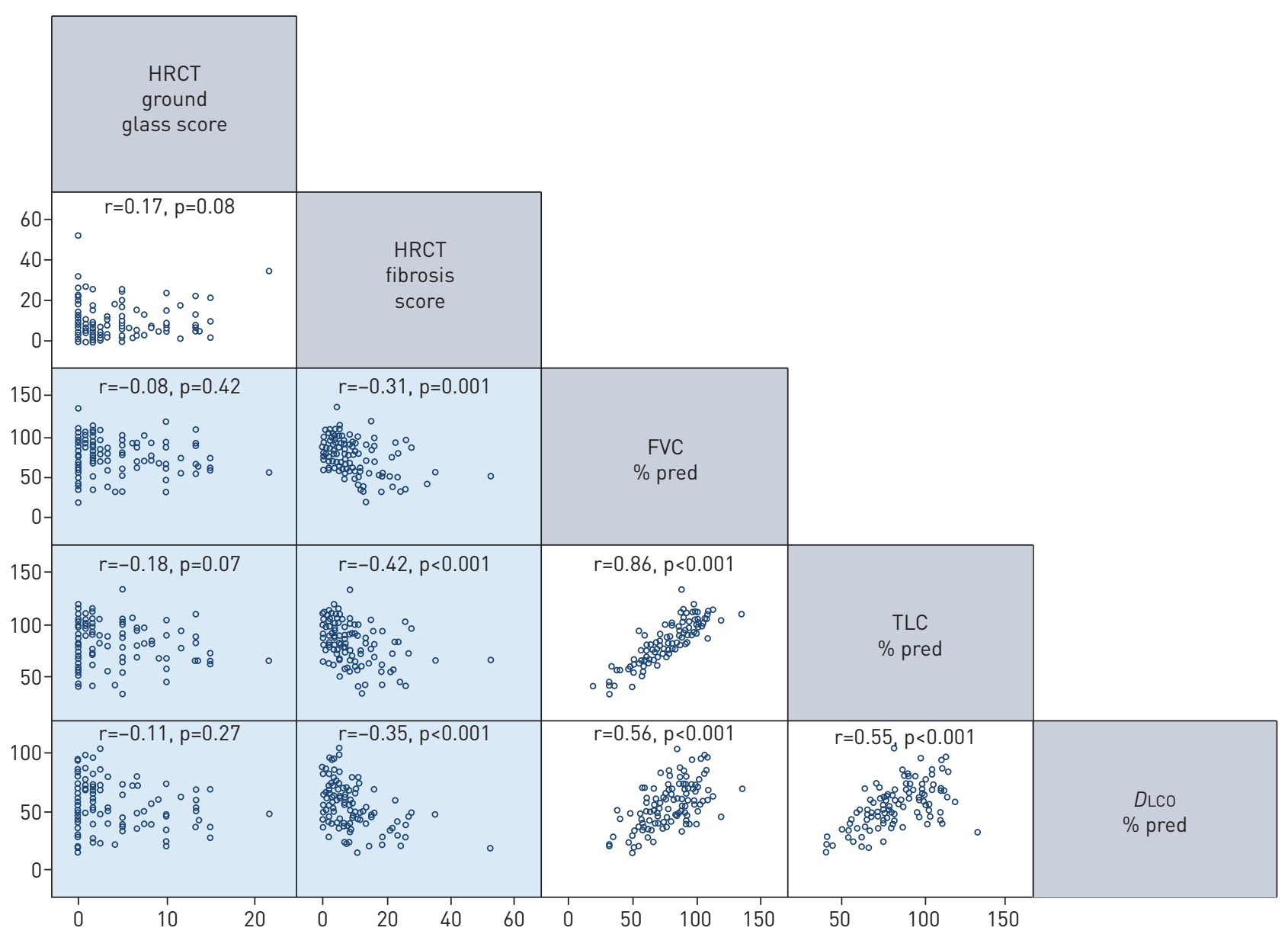

FIGURE 1 Correlation of lung function and high-resolution computed tomography (HRCT) parameters at baseline in the University of British Columbia cohort. Both $x$ and $y$ axes indicate \% predicted for lung function measurements and percent of affected lung for HRCT scores. Shaded scatterplots highlight the inconsistent association between lung function and HRCT measures. FVC: forced vital capacity; TLC: total lung capacity; DLCO: diffusing capacity of the lung for carbon monoxide.

systolic pressure (RVSP) $\geqslant 40 \mathrm{mmHg}$ by echocardiography was present in $12 \%$ of the UBC cohort at baseline. The median HRCT fibrosis score and total ILD scores were 6.7\% (3.3-10.8\%) and 10.0\% (4.2$17.5 \%)$ at baseline, respectively, and were highly correlated with each other $(\mathrm{r}=0.89, \mathrm{p}<0.001)$. Median survival was 11.0 years in the UBC cohort, with 39 patients (27\% of the cohort) deceased at the end of the follow-up period.

On unadjusted analysis, both HRCT fibrosis score and HRCT total ILD score were moderately associated with each lung function parameter in patients with SSc-ILD from the UBC cohort (figure 1). Independent predictors of HRCT fibrosis score and HRCT total ILD score included only total lung capacity (TLC) \% predicted and DLCO \% predicted, with an $\mathrm{R}^{2}$ of 0.20 for both models. HRCT ground glass score was not associated with any lung function measurement. Findings were similar when restricting the analysis to patients with an RVSP $<40 \mathrm{mmHg}$ and with exclusion of patients with $\geqslant 10$ pack-year smoking history or with anticentromere antibodies. In the UCSF cohort, HRCT total ILD score was associated with FVC \% predicted $(\mathrm{r}=-0.21, \mathrm{p}=0.05)$, TLC $\%$ predicted $(\mathrm{r}=0.28, \mathrm{p}=0.01)$, and DLCO $\%$ predicted $(\mathrm{r}=-0.42, \mathrm{p}<0.001)$ on unadjusted analysis. DLCO \% predicted was the only independent predictor of HRCT total ILD score in the UCSF cohort $\left(\mathrm{R}^{2}=0.11\right)$.

There was no association of any HRCT score with change in FVC, and only HRCT total ILD score was associated with change in DLCO. With adjustment for the ILD-GAP and SADL models, both HRCT fibrosis score and HRCT total ILD score were independently associated with survival, while there was no association of survival with HRCT ground glass score. The hazard ratio (HR) for the association of HRCT fibrosis score with mortality was 1.34 per $10 \%$ increase in fibrosis score (95\% CI 1.17-1.54) with adjustment for SADL $(\mathrm{p}=0.001)$, and $1.22(1.05-1.41)$ with adjustment for ILD-GAP $(\mathrm{p}=0.008)$. 
Although we identified both TLC and DLCO as independent predictors of HRCT severity, the strength of association was poor and HRCT severity was also not associated with change in lung function. Despite these findings, HRCT severity was an independent predictor of mortality with adjustment for established composite scores that are predominantly based on lung function. Together, these findings suggest that lung function and HRCT visual scores provide distinct and complementary information in patients with SSc-ILD.

Similar to previous studies in SSc-ILD [9-11], we confirm that DLCO is the physiological variable that is most strongly associated with radiological fibrosis severity; however, the multivariate models predicted only a minor amount of the variability in HRCT visual scores in both cohorts. This relatively poor model performance may relate to the presence of non-ILD manifestations of SSc that are captured by pulmonary function measurements, but not by HRCT. Importantly, the lack of strong association applied to all lung function measurements and persisted with exclusion of patients who had high RVSP, significant smoking history or anti-centromere antibodies. We replicated these findings in a second cohort. The slightly different HRCT scoring method used for our two cohorts is a strength of this study, indicating that our findings are less likely to be influenced by unique study population features or subjective radiologist interpretation.

We showed that HRCT visual scores were associated with mortality, including with adjustment for established prediction models, suggesting that HRCT severity might be useful as a tool for enrichment of clinical trials. These findings have important implications since FVC and DLCO have conventionally been used to monitor SSc-ILD patients, and FVC has also been used as the primary end-point in major SSc-ILD clinical trials [1, 2]. As recently recommended [12], our data suggest that clinical trials in SSc-ILD should include imaging-based analysis as a complementary and possibly more direct measurement of ILD severity. This is also supported by evidence that HRCT findings are more sensitive for monitoring disease progression in early SSc-ILD compared to FVC [13-15]. The small number of deaths in the UCSF cohort precluded validation of the mortality findings, and the non-standardised timing of HRCTs in our retrospective cohort also prevented a detailed assessment of how HRCT variables change over time and whether this change is prognostically informative. These are additional essential steps in demonstrating potential utility of HRCT variables in future clinical trials. Further studies are also needed to evaluate the relative merits of quantitative imaging compared to visual HRCT assessment.

In summary, we show that lung function is poorly associated with HRCT visual scores in two independent cohorts of patients with SSc-ILD, while HRCT severity is independently associated with future mortality. These findings demonstrate that pulmonary function tests and visual HRCT parameters are important and distinct measures in SSc-ILD, indicating that pulmonary function tests should be supplemented with additional ILD severity measures when precise determination of disease progression is desired.

Amrit Jhajj ${ }^{1,9}$, Harinder Pal Gill, ${ }^{1,9}$, Cameron J. Hague ${ }^{2}$, Darra Murphy ${ }^{2}$, Brett Elicker, Jeanette Soon ${ }^{2}$, Nada Sulaiman ${ }^{2}$, Daniela Castillo-Saldana ${ }^{1,4}$, Pearce G. Wilcox ${ }^{1}$, James Dunne ${ }^{1}$, Joyce S. Lee ${ }^{5}$, Paul J. Wolters ${ }^{6}$, Brett Ley $^{6}$, Kirk D. Jones ${ }^{7}$, Julie Morisset ${ }^{8}$ and Christopher J. Ryerson ${ }^{1,4}$

${ }^{1}$ Dept of Medicine, University of British Columbia, Vancouver, BC, Canada. ${ }^{2}$ Dept of Radiology, University of British Columbia, Vancouver, BC, Canada. ${ }^{3}$ Dept of Radiology, University of California San Francisco, San Francisco, CA, USA. ${ }^{4}$ Centre for Heart Lung Innovation, University of British Columbia, Vancouver, BC, Canada. ${ }^{5}$ Dept of Medicine, University of Colorado, Denver, CO, USA. ${ }^{6}$ Dept of Medicine, University of California San Francisco, San Francisco, CA, USA. ${ }^{7}$ Dept of Pathology, University of California San Francisco, San Francisco, CA, USA. ${ }^{8}$ Dept of Medicine, Centre Hospitalier de l'Université de Montréal, Montreal, QC, Canada. ${ }^{9}$ Contributed equally to this work.

Correspondence: Christopher J. Ryerson, St. Paul's Hospital, 1081 Burrard St, Ward 8B, Vancouver, BC, Canada V6Z 1Y6. E-mail: chris.ryerson@hli.ubc.ca

Received: Nov 162018 | Accepted after revision: Jan 312019

Conflict of interest: A. Jhajj has nothing to disclose. H.P. Gill has nothing to disclose. C.J. Hague has nothing to disclose. D. Murphy has nothing to disclose. B. Elicker has nothing to disclose. J. Soon has nothing to disclose. N. Sulaiman has nothing to disclose. D. Castillo-Saldana has nothing to disclose. P.G. Wilcox has nothing to disclose. J. Dunne has nothing to disclose. J.S. Lee reports grants from NIH, personal fees for advisory board work from Genentech, Boehringer Ingelheim and Celgene, outside the submitted work. P.J. Wolters reports grants from Genentech, grants and personal fees from Boehringer Ingelheim, outside the submitted work. B. Ley has nothing to disclose. K.D. Jones has nothing to disclose. J. Morisset reports personal fees from Roche and Boehringer Ingelheim, outside the submitted work. C.J. Ryerson reports grants and personal fees from Boehringer Ingelheim and Hoffmann-La Roche, outside the submitted work

Support statement: C.J. Ryerson is supported by a Career Investigator Award from the Michael Smith Foundation for Health Research. 


\section{References}

1 Tashkin DP, Elashoff R, Clements PJ, et al. Cyclophosphamide versus placebo in scleroderma lung disease. $N$ Engl J Med 2006; 354: 2655-2666.

2 Tashkin DP, Roth MD, Clements PJ, et al. Mycophenolate mofetil versus oral cyclophosphamide in scleroderma-related interstitial lung disease (SLS II): a randomised controlled, double-blind, parallel group trial. Lancet Respir Med 2016; 4: 708-719.

3 van den Hoogen F, Khanna D, Fransen J, et al. 2013 classification criteria for systemic sclerosis: an American College of Rheumatology/European League against Rheumatism collaborative initiative. Arthritis Rheum 2013; 65: 2737-2747.

4 Preliminary criteria for the classification of systemic sclerosis (scleroderma). Subcommittee for scleroderma criteria of the American Rheumatism Association Diagnostic and Therapeutic Criteria Committee. Arthritis Rheum 1980; 23: $581-590$.

5 Bourbeau J, Tan WC, Benedetti A, et al. Canadian Cohort Obstructive Lung Disease (CanCOLD): fulfilling the need for longitudinal observational studies in COPD. COPD 2014; 11: 125-132.

6 Goh NS, Desai SR, Veeraraghavan S, et al. Interstitial lung disease in systemic sclerosis: a simple staging system. Am J Respir Crit Care Med 2008; 177: 1248-1254.

7 Morisset J, Vittinghoff E, Elicker BM, et al. Mortality risk prediction in scleroderma-related interstitial lung disease: the SADL model. Chest 2017; 152: 999-1007.

8 Ryerson CJ, Vittinghoff E, Ley B, et al. Predicting survival across chronic interstitial lung disease: the ILD-GAP model. Chest 2014; 145: 723-728.

9 Ninaber MK, Stolk J, Smit J, et al. Lung structure and function relation in systemic sclerosis: application of lung densitometry. Eur J Radiol 2015; 84: 975-979.

10 Salaffi F, Carotti M, Bosello S, et al. Computer-aided quantification of interstitial lung disease from high resolution computed tomography images in systemic sclerosis: correlation with visual reader-based score and physiologic tests. Biomed Res Int 2015; 2015: 834262.

11 Goldin JG, Lynch DA, Strollo DC, et al. High-resolution CT scan findings in patients with symptomatic scleroderma-related interstitial lung disease. Chest 2008; 134: 358-367.

12 Khanna D, Seibold J, Goldin J, et al. Interstitial lung disease points to consider for clinical trials in systemic sclerosis. Rheumatology (Oxford) 2017; 56: Suppl. 5, v27-v32.

13 Wangkaew S, Euathrongchit J, Wattanawittawas P, et al. Correlation of delta high-resolution computed tomography (HRCT) score with delta clinical variables in early systemic sclerosis (SSc) patients. Quant Imaging Med Surg 2016; 6: 381-390.

14 Suliman YA, Dobrota R, Huscher D, et al. Brief report: pulmonary function tests: high rate of false-negative results in the early detection and screening of scleroderma-related interstitial lung disease. Arthritis Rheumatol 2015; 67: 3261-3256.

15 Afeltra A, Zennaro D, Garzia P, et al. Prevalence of interstitial lung involvement in patients with connective tissue diseases assessed with high-resolution computed tomography. Scand J Rheumatol 2006; 35: 388-394. 